\title{
ON LEGENDRIAN SINGULARITIES
}

\author{
SHYŪICHI IZUMIYA
}

(Communicated by Haynes R. Miller)

\begin{abstract}
We introduce three natural equivalence relations among Legendrian singularities and study their properties by using recent results of the theory of singularities of smooth map germs.
\end{abstract}

1. Introduction. The notion of Legendrian singularities has been introduced by V. I. Arnol'd in order to describe the notion of the wave front set $[\mathbf{1}, \mathbf{9}]$. In this paper we shall introduce three natural equivalence relations among Legendrian singularities and study their properties.

Let $N$ be a $(2 n+1)$-dimensional smooth manifold and $K$ be a contact structure on $N$ (i.e., $K$ is a nondegenerate tangent hyperplane field on $N$ ). An immersion $i: L \rightarrow N$ is said to be Legendrian if $\operatorname{dim} L=n$ and $d i_{x}\left(T_{x} L\right) \subset K_{x}$ for any $x \in L$. We say that a smooth fibre bundle $\pi: E \rightarrow M$ is Legendrian if its total space $E$ is furnished with a contact structure and its fibres are Legendrian submanifolds.

DEFINITION 1.1. Let $\pi: E \rightarrow M$ be a Legendrian bundle. For a Legendrian immersion $i: L \rightarrow E, \pi \circ i: L \rightarrow M$ is said to be a Legendrian map. The image of Legendrian map $\pi \circ i$ is said to be a wave front set of $i$. It is denoted by $W(i)$.

Let $f:(N, x) \rightarrow(P, y)$ denote the map germ at $x$ such that $f(x)=y$. We now introduce three natural equivalence relations.

DEFINITION 1.2. Let $\pi: E \rightarrow M$ be a Legendrian bundle. Let $i:(L, p) \rightarrow(E, q)$ and $i^{\prime}:\left(L^{\prime}, p^{\prime}\right) \rightarrow\left(E, q^{\prime}\right)$ be Legendrian immersion germs. Then we say that

(1) $i$ and $i^{\prime}$ are wave front equivalent if there exists a diffeomorphism $h:(M, \pi(q))$ $\rightarrow\left(M, \pi\left(q^{\prime}\right)\right)$ such that $h(W(i))=W\left(i^{\prime}\right)$ as set germs.

(2) $i$ and $i^{\prime}$ are weak Legendrian equivalent if there exist diffeomorphism germs $h:(L, p) \rightarrow\left(L^{\prime}, p^{\prime}\right)$ and $H:(M, \pi(q)) \rightarrow\left(M, \pi\left(q^{\prime}\right)\right)$ such that $H \circ(\pi \circ i)=\left(\pi \circ i^{\prime}\right) \circ h$.

(3) $i$ and $i^{\prime}$ are Legendrian equivalent if there exist diffeomorphism germs $h:(L, p)$ $\rightarrow\left(L^{\prime}, p^{\prime}\right)$ and a contact diffeomorphism germ $H:(E, q) \rightarrow\left(E, q^{\prime}\right)$ which sends fibres to fibres such that $H \circ i=i^{\prime} \circ h$.

Legendrian equivalence has been studied by V. I. Arnol'd and V. M. Zakalyukin, using their classification theory of function germs $[\mathbf{1}, \mathbf{9}]$. We shall apply recent results of the theory of singularities of smooth map germs in $[\mathbf{2}, \mathbf{4}, \mathbf{5}, \mathbf{6}, \mathbf{7}]$ to these equivalence relations.

DEFINITION 1.3. A Legendrian map germ at a point is said to be Legendrian stable if for every map with the given germ there is a neighborhood in the space of Legendrian maps (in the $C^{\infty}$-topology) and a neighborhood of the original point such that each Legendrian map belonging to the first neighborhood has in the

Received by the editors April 9, 1986.

1980 Mathematics Subject Classification (1985 Revision). Primary 58C27; Secondary 57R45.

Key words and phrases. Legendrian singularities, wave front. 
second neighborhood a point at which its germ is Legendrian equivalent to the original germ.

We now state the main theorem in this paper.

THEOREM 1.4. Let $\pi: E \rightarrow M$ be a Legendrian bundle. Let $i:(L, p) \rightarrow(E, q)$ and $i^{\prime}:\left(L^{\prime}, p^{\prime}\right) \rightarrow\left(E, q^{\prime}\right)$ be Legendrian immersion germs such that $i$ and $i^{\prime}$ are Legendrian stable. Then the following are equivalent:

(1) $i$ and $i^{\prime}$ are wave front equivalent.

(2) $i$ and $i^{\prime}$ are weak Legendrian equivalent.

(3) $i$ and $i^{\prime}$ are Legendrian equivalent.

By the result of Zakalyukin [9], generic Legendrian singularities are Legendrian stable if $\operatorname{dim} M \leq 6$. It is important to study topological classification theory in the case where $\operatorname{dim} M \geq 7$. See [3].

All map germs and diffeomorphisms considered here are differentiable of class $C^{\infty}$, unless stated otherwise.

2. Generating families (including a quick review of Arnol'd-Zakalyukin theory). Legendrian manifolds are bound up with hypersurfaces and Legendrian singularities with singularities of families of hypersurfaces $[\mathbf{1}, \mathbf{9}]$. In this section we shall describe Legendrian immersion germs in terms of generalized phase function germs analogous to the survey article of C. T. C. Wall [8]. Let $\pi: E \rightarrow$ $M$ be a Legendrian bundle. For any $p \in E$, there is a local coordinate system $\left(x_{1}, \ldots, x_{m}, y_{1}, \ldots, y_{m}, z\right)$ about $p$ such that $\pi\left(x_{1}, \ldots, x_{m}, y_{1}, \ldots, y_{m}, z\right)=$ $\left(x_{1}, \ldots, x_{m}, z\right)$ and the contact structure is given by the 1 -form $\alpha=d z-\sum_{i=1}^{m} y_{i} d x_{i}$ $[1,20.3]$. Hence, we shall only consider the standard Legendrian bundle $\pi: \mathbf{R}^{2} m+1 \rightarrow$ $\mathbf{R}^{m} \times \mathbf{R}$.

Let $F:\left(\mathbf{R}^{m} \times \mathbf{R}^{k}, 0\right) \rightarrow(\mathbf{R}, 0)$ be a map germ such that $d_{2} F$ is nonsingular at 0 . Here, $d_{2} F:\left(\mathbf{R}^{m} \times \mathbf{R}^{k}, 0\right) \rightarrow\left(\mathbf{R}^{k}, 0\right)$ is given by $d_{2} F(q, x)=\left(\partial F / \partial x_{1}, \ldots, \partial F / \partial x_{k}\right)$. Then $F$ is called a generalized phase function germ. The critical set $C(F)=$ $d_{2} F^{-1}(0)$ is a smooth $m$-manifold. We can prove that the map germ $\Phi_{F}:(C(F), 0)$ $\rightarrow \mathbf{R}^{2 m+1}$ given by

$$
\Phi_{F}(q, x)=\left(q, \frac{\partial F}{\partial q}(q, x), F(q, x)\right)
$$

is a Legendrian immersion.

Then we have the following proposition.

PROPOSITION 2.1 (ARNOL'D-ZAKALYUKIN [1, 9]). Any Legendrian immersion germ is given by the above construction.

Let $F:\left(\mathbf{R}^{m} \times \mathbf{R}^{k}, 0\right) \rightarrow(\mathbf{R}, 0)$ be a generalized phase function germ. We define $\tilde{F}:\left(\mathbf{R}^{m} \times \mathbf{R} \times \mathbf{R}^{k}, 0\right) \rightarrow(\mathbf{R}, 0)$ by $\tilde{F}(q, z, x)=F(q, x)-z$. We call $\tilde{F}$ a generating family of $\Phi_{F}$.

Let $\mathcal{E}_{n}$ be the ring of function germs $\left(\mathbf{R}^{n}, 0\right) \rightarrow \mathbf{R}$ and $\mathfrak{M}_{n}$ be the unique maximal ideal. For each map germ $f:\left(\mathbf{R}^{n}, 0\right) \rightarrow\left(\mathbf{R}^{p}, 0\right)$, we define $f^{*}: E_{p} \rightarrow E_{n}$ by $f^{*}(h)=$ $h \circ f$.

DEFINITION 2.2. (1) Let $f, g:\left(\mathbf{R}^{n} \times \mathbf{R}^{k}, 0\right) \rightarrow(\mathbf{R}, 0)$ be function germs. We say that $f$ and $g$ are $P-K$-equivalent if there exists a diffeomorphism germ $\Psi$ : $\left(\mathbf{R}^{n} \times \mathbf{R}^{k}, 0\right) \rightarrow\left(\mathbf{R}^{n} \times \mathbf{R}^{k}, 0\right)$ of the form $\Psi(u, x)=\left(\psi(u), \Psi_{2}(u, x)\right)$ for $(u, x) \in$ $\mathbf{R}^{n} \times \mathbf{R}^{k}$ such that $\Psi^{*}\left(\langle f\rangle_{\varepsilon_{n+k}}\right)=\langle g\rangle_{\varepsilon_{n+k}}$. 
(2) Let $f:\left(\mathbf{R}^{n} \times \mathbf{R}^{k}, 0\right) \rightarrow(\mathbf{R}, 0)$ and $g:\left(\mathbf{R}^{n} \times \mathbf{R}^{k^{\prime}}, 0\right) \rightarrow(\mathbf{R}, 0)$ be function germs. We say that $f$ and $g$ are stably $P$-K -equivalent if they become $P$-K -equivalent after the addition of nondegenerate quadratic forms in additional variables.

The following theorem is very important.

THEOREM 2.3 (ARNOL'D-ZAKALYUKIN $[\mathbf{1}, \mathbf{9}])$. Let $\tilde{F}:\left(\left(\mathbf{R}^{m} \times \mathbf{R}\right) \times \mathbf{R}^{k}, 0\right) \rightarrow$ $(\mathbf{R}, 0)\left(\right.$ resp. $\left.\tilde{G}:\left(\left(\mathbf{R}^{m} \times \mathbf{R}\right) \times \mathbf{R}^{k^{\prime}}, 0\right) \rightarrow(\mathbf{R}, 0)\right)$ be a generating family of the Legendrian immersion germ $\Phi_{F}\left(\right.$ resp. $\left.\Phi_{G}\right)$. Then

(1) $\Phi_{F}$ and $\Phi_{G}$ are Legendrian equivalent if and only if $\tilde{F}$ and $\tilde{G}$ are stably $P-K$-equivalent.

(2) $\Phi_{F}$ is Legerdrian stable if and only if $\tilde{F}$ is a K-versal deformation of $F \mid 0 \times \mathbf{R}^{k}$. (For the definition of the $\mathcal{K}$-versal deformation, see [4].)

3. Proof of Theorem 1.4. In this section we prove Theorem 1.4, using results in $([\mathbf{2}, \mathbf{4}, \mathbf{5}, \mathbf{6}, \mathbf{7}])$. For this purpose, we need some notations.

Let $F, G:\left(\mathbf{R}^{m} \times \mathbf{R}^{k}, 0\right) \rightarrow(\mathbf{R}, 0)$ be function germs. We say that $\left(\pi_{m}, F\right)$ and $\left(\pi_{m}, G\right)$ are A-equivalent if there exist diffeomorphism germs $H:\left(\mathbf{R}^{m} \times \mathbf{R}^{k}, 0\right) \rightarrow$ $\left(\mathbf{R}^{m} \times \mathbf{R}^{k}, 0\right)$ and $K:\left(\mathbf{R}^{m} \times \mathbf{R}, 0\right) \rightarrow\left(\mathbf{R}^{m} \times \mathbf{R}, 0\right)$ such that $K \circ\left(\pi_{m}, F\right)=\left(\pi_{m}, G\right) \circ H$, where $\left(\pi_{m}, F\right)(q, x)=(q, F(q, x))$. The definition of stable A-equivalence is the same as that of stable $\mathcal{K}$-equivalence. For each map germ $f:\left(\mathbf{R}^{n}, 0\right) \rightarrow\left(\mathbf{R}^{p}, 0\right)$, we denote by $\theta(f)$ the $\varepsilon_{n}$-module of all vector field germs along $f$. We define an $\mathcal{E}_{n}$-homomorphism $t f: \theta(n) \rightarrow \theta(f)$ by $t f(\xi)=d f \circ \xi$, where $\theta(n)=\theta\left(1_{\mathbf{R}} n\right)$. We also define an $\mathcal{E}_{p}$-homomorphism $\omega f: \theta(p) \rightarrow \theta(f)$ by $\omega f(\eta)=\eta \circ f$.

We say that $f$ is $A$-stable if $\theta(f)=t f(\theta(n)+\omega f(\theta(p))$.

Then we have the following lemma.

LEMMA 3.1. Let $F:\left(\mathbf{R}^{m} \times \mathbf{R}^{k}, 0\right) \rightarrow(\mathbf{R}, 0)$ be a generalized phase function germ. If the Legendrian immersion germ $\Phi_{F}$ is Legendrian stable, then $\left(\pi_{m}, F\right)$ is A-stable.

ProOF. By Theorem 2.3, $\tilde{F}$ is a $K$-versal deformation of $f=F \mid 0 \times \mathbf{R}^{k}$, and it follows that

$$
\varepsilon_{k}=\left\langle\frac{\partial f}{\partial x_{1}}, \ldots, \frac{\partial f}{\partial x_{k}}, f\right\rangle_{\varepsilon_{k}}+\left\langle\frac{\partial F}{\partial q_{1}}\left|0 \times \mathbf{R}^{k}, \ldots, \frac{\partial F}{\partial q_{m}}\right| 0 \times \mathbf{R}^{k}, 1\right\rangle_{\mathbf{R}} .
$$

We can show that

$$
\mathcal{E}_{m+k}=\left\langle\frac{\partial F}{\partial x_{1}}, \ldots, \frac{\partial F}{\partial x_{k}}, F\right\rangle_{\mathcal{E}_{m+k}}+\left\langle\frac{\partial F}{\partial q_{1}}, \ldots, \frac{\partial F}{\partial q_{m}}, 1\right\rangle_{\varepsilon_{m}}+\mathfrak{M}_{m} \mathcal{E}_{m+k}
$$

By the Malgrange preparation theorem, we have

$$
\varepsilon_{m+k}=\left\langle\frac{\partial F}{\partial x_{1}}, \ldots, \frac{\partial F}{\partial x_{k}}, F\right\rangle_{\varepsilon_{m+k}}+\left\langle\frac{\partial F}{\partial q_{1}}, \ldots, \frac{\partial F}{\partial q_{m}}, 1\right\rangle_{\varepsilon_{m}} .
$$
that

Since we have a decomposition $\theta\left(\left(\pi_{m}, F\right)\right)=\theta\left(\pi_{m}\right) \oplus \mathcal{E}_{m+k}$, then we can show

$$
\begin{aligned}
\theta\left(\left(\pi_{m}, F\right)\right)= & t\left(\pi_{m}, F\right)(\theta(m+k))+\omega\left(\pi_{m}, F\right)(\theta(m+1)) \\
& +\left(\pi_{m}, F\right)^{*}\left(\mathfrak{M}_{m+1}\right) \theta\left(\left(\pi_{m}, F\right)\right) .
\end{aligned}
$$

Applying the Malgrange preparation theorem once again, we have

$$
\theta\left(\left(\pi_{m}, F\right)\right)=t\left(\pi_{m}, F\right)(\theta(m+k))+\omega\left(\pi_{m}, F\right)(\theta(m+1)) .
$$


CoRollaRY 3.2. Let $F:\left(\mathbf{R}^{m} \times \mathbf{R}^{k}, 0\right) \rightarrow(\mathbf{R}, 0)$ and $G:\left(\mathbf{R}^{m} \times \mathbf{R}^{k^{\prime}}, 0\right) \rightarrow(\mathbf{R}, 0)$ be generalized phase function germs such that $\Phi_{F}$ and $\Phi_{G}$ are Legendrian stable. Then the following are equivalent:

(1) $\Phi_{F}$ and $\Phi_{G}$ are Legendrian equivalent.

(2) $\left(\pi_{m}, F\right)$ and $\left(\pi_{m}, G\right)$ are stably A-equivalent.

PROOF. It is enough to prove the statement in the case where $k=k^{\prime}$. We put $f=F \mid 0 \times \mathbf{R}^{k}$; then there is a canonical isomorphism $Q_{m+2}\left(\pi_{m}, F\right) \cong Q_{m+2}(f)$, where

$$
Q_{m+2}\left(\pi_{m}, F\right)=\mathcal{E}_{m+k} /\left(\pi_{m}, F\right)^{*}\left(\mathfrak{M}_{m+1}\right) \mathcal{E}_{m+k}+\mathfrak{M}_{m+k}^{m+3}
$$

and

$$
Q_{m+2}(f)=\mathcal{E}_{k} / f^{*}\left(\mathfrak{M}_{1}\right) \mathcal{E}_{k}+\mathfrak{M}_{k}^{m+3} .
$$

It follows that $f$ and $g=G \mid 0 \times \mathbf{R}^{k}$ are $\mathcal{K}$-equivalent if and only if $\left(\pi_{m}, F\right)$ and $\left(\pi_{m}, G\right)$ are $\mathcal{K}$-equivalent [5]. Since $\Phi_{F}$ and $\Phi_{G}$ are Legendrian stable, then $\tilde{F}$ and $\tilde{G}$ are respectively $\mathcal{K}$-versal deformations of $f$ and $g$. By the uniqueness of the $\mathcal{K}$-versal deformation, $\tilde{F}$ and $\tilde{G}$ are $P$-K -equivalent if and only if $f$ and $g$ are $K$ equivalent. On the other hand, by the classification theorem of $A$-stable map germs in [6], $\left(\pi_{m}, F\right)$ and $\left(\pi_{m}, G\right)$ are $A$-equivalent if and only if $\left(\pi_{m}, F\right)$ and $\left(\pi_{m}, G\right)$ are $K$-equivalent. This completes the proof.

We have the following simple lemma.

LEMMA 3.3. Let $F:\left(\mathbf{R}^{m} \times \mathbf{R}^{k}, 0\right) \rightarrow(\mathbf{R}, 0)$ be a generalized phase function germ. Then $C(F)=\Sigma\left(\pi_{m}, F\right)$ and $\pi \circ \Phi_{F}=\left(\pi_{m}, F\right) \mid \Sigma\left(\pi_{m}, F\right)$. Here $\pi: \mathbf{R}^{2 m+1} \rightarrow$ $\mathbf{R}^{m} \times \mathbf{R}$ is the standard Legendrian bundle and $\Sigma\left(\pi_{m}, F\right)$ is the critical set (germ) of $\left(\pi_{m}, F\right)$.

We now refer to notions and results in [7].

Definition 3.4 [7, Definition (0.8)]. A map germ $f:\left(\mathbf{R}^{n}, 0\right) \rightarrow\left(\mathbf{R}^{p}, 0\right)$ has nondegenerate critical set if

(1) $J(f)=I(\Sigma(f))$

(2) $\operatorname{dim} \Sigma(f)=p-1$ in $\mathbf{R}^{n}$.

Here $J(f)$ is the Jacobian ideal of $f$ and $I(X)=\left\{\phi \in E_{n} \mid \phi\right.$ vanishes on $\left.X \subset \mathbf{R}^{n}\right\}$.

In [7], du Plessis and Wilson proved the following result.

THEOREM $3.5\left[\mathbf{7}\right.$, Theorem (0.14)]. Let $f:\left(\mathbf{R}^{n}, 0\right) \rightarrow\left(\mathbf{R}^{p}, 0\right)$ be a map germ with nondegenerate critical set. Let $g:\left(\mathbf{R}^{n}, 0\right) \rightarrow\left(\mathbf{R}^{p}, 0\right)$ be a map germ satisfying

( $\alpha) \Sigma(f)=\Sigma(g)$

( $\beta) f|\Sigma(f)=g| \Sigma(g)$,

$(\gamma)$ Image $d g_{x} \subset$ Image $d f_{x}$ for $x$ in some dense subset of $\Sigma(f)$.

Suppose that $f$ has rank 1 and zero full second intrinsic derivative. Then $f$ and $g$ are right equivalent. Here, $f$ and $g$ are right equivalent if there exists a diffeomorphism germ $h:\left(\mathbf{R}^{n}, 0\right) \rightarrow\left(\mathbf{R}^{n}, 0\right)$ such that $f=g \circ h$.

PROOF OF (3) FROM (2). By Proposition 2.1, we only consider the case where Legendrian immersion germs are given by generalized phase function germs. Let $F:\left(\mathbf{R}^{m} \times \mathbf{R}^{k}, 0\right) \rightarrow(\mathbf{R}, 0)$ and $G:\left(\mathbf{R}^{m} \times \mathbf{R}^{k^{\prime}}, 0\right) \rightarrow(\mathbf{R}, 0)$ be generalized phase function germs. By Thom's splitting lemma, we may assume that $F \mid 0 \times \mathbf{R}^{k}$ has no quadratic part and $k=k^{\prime}$. By Lemma 3.3, $\Phi_{F}$ and $\Phi_{G}$ are weak Legendrian 
equivalent if and only if there exist diffeomorphism germs $K:\left(\mathbf{R}^{m} \times \mathbf{R}^{k}, 0\right) \rightarrow$ $\left(\mathbf{R}^{m} \times \mathbf{R}^{k}, 0\right)$ and $H:\left(\mathbf{R}^{m} \times \mathbf{R}, 0\right) \rightarrow\left(\mathbf{R}^{m} \times \mathbf{R}, 0\right)$ such that

$K\left(\Sigma\left(\pi_{m}, F\right)\right)=\Sigma\left(\pi_{m}, G\right) \quad$ and $\quad\left(\pi_{m}, G\right) \circ K\left|\Sigma\left(\pi_{m}, F\right)=H \circ\left(\pi_{m}, F\right)\right| \Sigma\left(\pi_{m}, F\right)$.

We now put $G^{\prime}=\left(\pi_{m}, G\right) \circ K$ and $F^{\prime}=H \circ\left(\pi_{m}, F\right)$, then $\Sigma\left(G^{\prime}\right)=\Sigma\left(F^{\prime}\right)=$ $\Sigma\left(\pi_{m}, F\right)$ and $G^{\prime}\left|\Sigma\left(G^{\prime}\right)=F^{\prime}\right| \Sigma\left(F^{\prime}\right)$. If we check that $F^{\prime}$ and $G^{\prime}$ satisfy the assumption in Theorem 3.5, then the proof will be completed by Corollary 3.2. Since $\operatorname{rank}\left(\pi_{m}, F\right)=m$ and $F \mid 0 \times \mathbf{R}^{k}$ has no quadratic part, it is enough to check that $F^{\prime}$ has nondegenerate critical set and $F^{\prime}, G^{\prime}$ satisfy the condition $(\gamma)$. Because $\Sigma\left(\pi_{m}, F\right)$ is an $m$-dimensional smooth manifold and $\left(\pi_{m}, F\right)$ is $A$-stable, $\left(\pi_{m}, F\right) \mid \Sigma\left(\pi_{m}, F\right)$ is an immersion on a dense subset of $\Sigma\left(\pi_{m}, F\right)$. The rank of $\left(\pi_{m}, F\right) \mid \Sigma\left(\pi_{m}, F\right)$ is equal to $m$ at such a point $x \in \Sigma\left(\pi_{m}, F\right)$. Then we have Image $d\left(F^{\prime} \mid \Sigma\left(F^{\prime}\right)\right)_{x}=$ Image $d F_{x}^{\prime}$ for $x$ in some dense subset of $\Sigma\left(F^{\prime}\right)$. On the other hand, since $G^{\prime}\left|\Sigma\left(G^{\prime}\right)=F^{\prime}\right| \Sigma\left(F^{\prime}\right)$, we have Image $d\left(G^{\prime} \mid \Sigma\left(G^{\prime}\right)\right)_{x}=$ Image $d\left(F^{\prime} \mid \Sigma\left(F^{\prime}\right)\right)_{x}$. Hence, Image $d\left(G^{\prime} \mid \Sigma\left(G^{\prime}\right)\right)_{x}=$ Image $d G_{x}^{\prime}$ for $x$ in the same dense subset of $\Sigma\left(F^{\prime}\right)=\Sigma\left(G^{\prime}\right)$ as the above.

Because $d_{2} F:\left(\mathbf{R}^{m} \times \mathbf{R}^{k}, 0\right) \rightarrow\left(\mathbf{R}^{k}, 0\right)$ is a submersion germ, we have

$$
I\left(\Sigma\left(\pi_{m}, F\right)\right)=I\left(d_{2} F^{-1}(0)\right)=\left\langle\frac{\partial F}{\partial x_{1}}, \ldots, \frac{\partial F}{\partial x_{k}}\right\rangle_{\varepsilon_{m+k}}=J\left(\pi_{m}, F\right) .
$$

Then $F^{\prime}=H \circ\left(\pi_{m}, F\right)$ has nondegenerate critical set.

The proof of (2) from (1) is a direct application of the $C^{\infty}$-normalization theorem in [2]. This completes the proof of Theorem 1.4.

REMARK. Let $F:\left(\mathbf{R}^{m} \times \mathbf{R}^{k}, 0\right) \rightarrow(\mathbf{R}, 0)$ be a generalized phase function germ. By the proof of Corollary 3.2, the Legendrian equivalence class of the stable Legendrian immersion $\Phi_{F}$ is completely determined by the "stably" isomorphism class of R-algebra $Q_{m+2}\left(\pi_{m}, F\right)$.

\section{REFERENCES}

1. V. I. Arnol'd, S. M. Gusein-Zade, and A. N. Varchenko, Singularities of differentiable maps, vol. 1, Monographs in Math. 82, Birkhäuser, 1985.

2. T. Gaffney and L. Wilson, Equivalence of generic mappings and $C^{\infty}$ normalization, Compositio Math. 49 (1983), 291-308.

3. S. Izumiya, A topological property of Legendrian singularities, in preparation.

4. J. Martinet, Déploîments versal des applications différentiables et classification des applications stables, Singularités d'Applications Différentiables, Lecture Notes in Math., vol. 535, Springer-Verlag, Berlin and New York, 1976, pp. 11-44.

5. J. Mather, Stability of $C^{\infty}$ mappings. III: Finitely determined map germs, Inst. Hautes Études Sci. Publ. Math. 35 (1969), 127-156.

6. $\ldots$, Stability of $C^{\infty}$ mappings. IV: Classification of stable germs by $\mathbf{R}$-algebras, Inst. Hautes Études Sci. Publ. Math. 37 (1970), 223-248.

7. A. du Plessis and L. Wilson, On right-equivalence, Math. Z. 190 (1985), 163-205.

8. C. T. C. Wall, Geometric property of generic differentiable manifolds, Geometry and Topology III, Lecture Notes in Math., vol. 597, Springer-Verlag, Berlin and New York, 1977, pp. 707774.

9. V. M. Zakalyukin, Lagrangian and Legendrian singularities, Funct. Anal. Appl. 10 (1976), 37-45.

Department of Mathematics, Faculty of Science, Hokkaido University, SAPPORO 060, JAPAN 